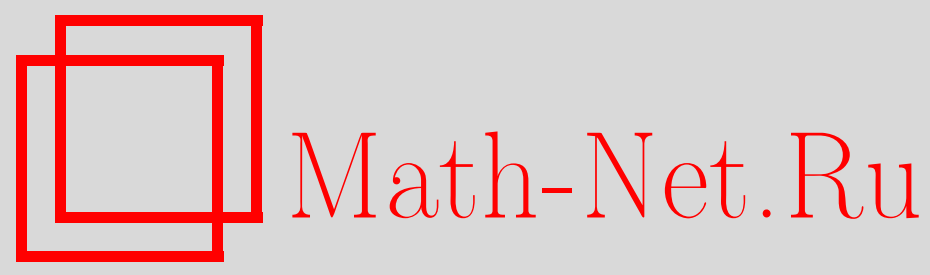

А. Р. Алимов, Геометрическая характеризация строгих солнц в пространстве $\ell^{\infty}(n)$, Матем. заметки, 2001, том 70, выпуск 1, 3-11

DOI: https://doi.org/10.4213/mzm712

Использование Общероссийского математического портала Math-Net.Ru подразумевает, что вы прочитали и согласны с пользовательским соглашением http://www.mathnet.ru/rus/agreement

Параметры загрузки:

IP: 52.87 .193 .239

26 апреля 2023 г., 08:13:23

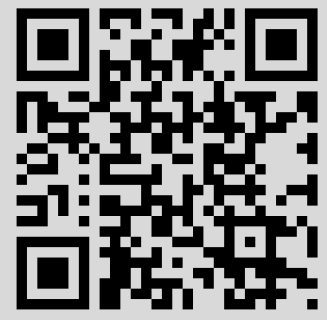




\section{ГЕОМЕТРИЧЕСКАЯ ХАРАКТЕРИЗАЦИЯ СТРОГИХ СОЛНЦ В ПРОСТРАНСТВЕ $\ell^{\infty}(n)$}

\section{А.Р. Алимов}

Подмножество $M$ линейного нормированного пространства $X$ называется $c$ трогим солнием, если для каждой точки $x \in X \backslash M$ множество ее ближайших точек из $M$ непусто и для каждой точки $y \in M$, ближайшей к $x$, точка $y$ является ближайшей точкой из $M$ для всех точек луча $\{\lambda x+(1-\lambda) y \mid \lambda>0\}$. В статье получена геометрическая характеризация строгих солнц в пространстве $\ell^{\infty}(n)$.

Библиограффия: 17 названий.

Подмножество $M$ линейного нормированного пространства $X$ назьвается $с т р о г и м$ солнием, если для каждой точки $x \in X \backslash M$ множество ее ближайших точек из $M$ непусто и для каждой точки $y \in M$, ближайшей к $x$, точка $y$ является ближайшей точкой из $M$ для всех точек луча, начинающегося в $y$ и проходящего через $x$. Строгое солнще всегда непусто и замкнуто (о строгих солнщах см. [1]-[3]).

Для точки $x \in X$ посредством $P_{M} x$ обозначается множество ее ближайших элементов из $M$, т.е.

$$
P_{M} x=\left\{y \in M \mid\|x-y\|=\rho(x, M):=\inf _{z \in M}\|x-z\|\right\} .
$$

В данной работе получена внутренняя геометрическая характеризация строгих солнц в пространстве $\ell^{\infty}(n), n \in \mathbb{N}$.

Подмножество $M \subset \mathbb{R}^{n}$ называется $\ell^{1}$-выпукльм. , если для всех $x, y \in M, x \neq y$, найдется точка $z \in M, z \neq x, z \neq y$, такая, что $\|x-y\|_{\ell^{1}}=\|x-z\|_{\ell^{1}}+\|z-y\|_{\ell^{1}}$ (здесь $\|\cdot\|_{\ell^{1}}-$ стандартная $\ell^{1}$-норма на $\mathbb{R}^{n}$ ). Слово “вьпукло" в данном контексте было впервые использовано Менгером [4] (см. [5]). Так как $\ell^{1}$-норма не строго вьпукла, то векторы $x-y$ и $x-z$ не обязаны быть коллинеарными.

Для множества $M \subset \mathbb{R}^{n}$ определим

$$
\begin{gathered}
\operatorname{eqc}(M)=\operatorname{eqc}_{\mathbb{R}^{n}}(M):=\left\{j \in\{1, \ldots, n\} \mid x_{j}=y_{j} \text { для всех } x, y \in M\right\}, \\
x=\left(x_{1}, \ldots, x_{n}\right), \quad y=\left(y_{1}, \ldots, y_{n}\right) ;
\end{gathered}
$$

"eqc" - аббревиатура выражения "equal coordinates" - равные координаты.

Для $x, y \in \mathbb{R}^{n}$ и $M \subset \mathbb{R}^{n}$ мы пишем, что

$x \neq_{м} y$, если $x_{j} \neq y_{j}$ для всех $j \notin \operatorname{eqc}(M)$;

$x \neq y$, если $x_{i} \neq y_{i}$ для всех $i=1, \ldots, n$.

Работа выполнена при поддержке программ "Ведущие научные школы”, грант № 96-15-96102, и Российского фонда фундаментальных исследований, грант № 99-01-00357. 
Мы называем множество $M$ строго $\ell^{1}$-выпуклым, если

1) оно $\ell^{1}$-выпукло и

2 ) для всех $x, y \in M, x \neq_{M} y$, найдется $\ell^{1}$-геодезический сегмент $k(t) \subset M, 0 \leqslant t \leqslant 1$, соединяющий $x$ и $y$, такой, что его координатные функции $[0,1] \ni t \mapsto k_{j}(t)$ монотонны для $j=1,2, \ldots, n$ и строго монотонны для $j \notin \operatorname{eqc}(M)$.

(Под $\ell^{1}$-геодезическим сегментом мы подразумеваем дугу длины $\|x-y\|_{\ell^{1}}$, начинающуюся в $x$ и заканчивающуюся в $y$.) В данных терминах $\ell^{1}$-выпуклость множества $M$ означает [6], что любые две точки $x, y \in M$ могут быть соединены $\ell^{1}$-геодезическим сегментом $k(\cdot) \subset M$, координатные функции $k_{i}(t)$ которого монотонны по $t$ (но не обязательно строго монотонны).

На рис. 1 даны примеры $\ell^{1}$-вьпуклых множеств из $\mathbb{R}^{2}$ и $\mathbb{R}^{3}$. Строго $\ell^{1}$-выпукльги из них являются лишь множества во второй и четвертой системе координат.
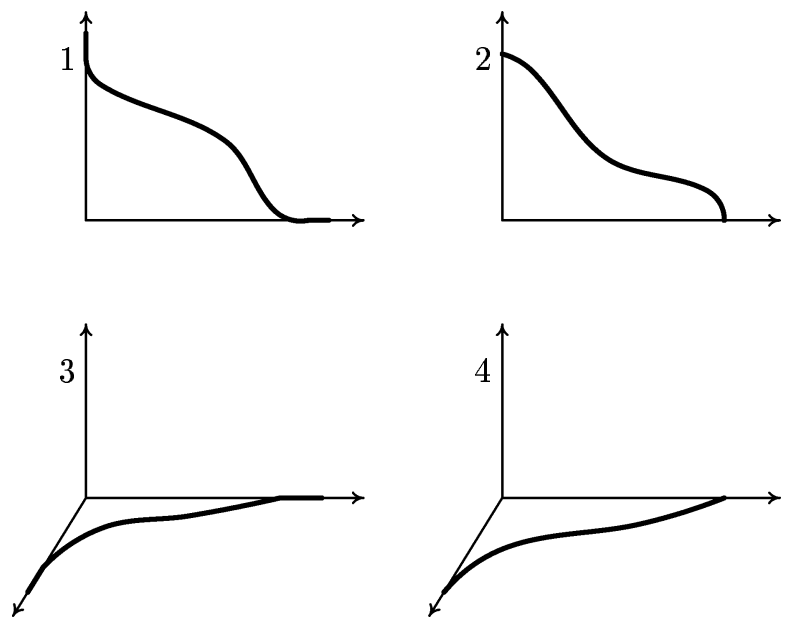

РИС. 1

Свойство строгой $\ell^{1}$-вьпуклости (как и свойство $\ell^{1}$-выпуклости) является наследственньм. Именно, непосредственно из определения следует, что если $M \subset \mathbb{R}^{k} \subset \mathbb{R}^{m}-$ строго $\ell^{1}$-выпуклое множество в $\mathbb{R}^{k}$, то оно строго $\ell^{1}$-выпукло и в $\mathbb{R}^{m}$. Если множество $M \ell^{1}$-выпукло в $\mathbb{R}^{m}$ и $k \leqslant m$, то $M \subset \mathbb{R}^{k} \ell^{1}$-вьпукло в $\mathbb{R}^{k}$.

Однако при переходе к меньшей размерности свойство строгой $\ell^{1}$-вьпуклости может теряться, хотя $\ell^{1}$-вьпуклость сохраняется всегда. Действительно, пусть $A=(2,0,0)$, $B=(0,2,0) \in \mathbb{R}^{3}$. Для $0 \leqslant t \leqslant 1$ определим точку $C_{t}=(t, t, 0)$ и построим семейство однозвенных ломаных в плоскости $\mathbb{R}^{2}=\{z=0\}$ :

$$
M_{t}=\left[A, C_{t}\right] \cup\left[C_{t}, B\right], \quad 0 \leqslant t<1, \quad M_{1}=[A, B] .
$$

Тогда множество

$$
M=\bigcup_{0 \leqslant t \leqslant 1}\left(M_{t}+(0,0, t)\right)
$$

строго $\ell^{1}$-выпукло в $\mathbb{R}^{3}$, но его сечение плоскостью $\mathbb{R}^{2}=\{z=0\}$ представляет собой объединение отрезков $[A, 0]$ и $[0, B]$ и не является строго $\ell^{1}$-выпуклым множеством в $\mathbb{R}^{2}$. Однако, по основной теореме (ниже) будет следовать, что $M-$ строгое солнце в $\ell^{\infty}(3)$. 
Под основным кокрестом в $\mathbb{R}^{n}$ мы будем понимать любое из множеств вида

$$
c\left(\mathbb{R}^{n}\right)=\left\{x \in \mathbb{R}^{n} \mid x_{j}=\operatorname{const}(j) \text { для некоторого } j \in\{1, \ldots, n\}\right\}, \quad \operatorname{eqc}\left(c\left(\mathbb{R}^{n}\right)\right)=\varnothing,
$$

(здесь const $(j)$ - константа, зависящая только от координаты $j$ ); а под кокрестом $c_{\mathbb{R}^{n}}(J)$ по набору координат $J=\left\{j_{1}, \ldots, j_{k}\right\} \subset\{1, \ldots, n\}$ мы будем понимать множество

$$
c_{\mathbb{R}^{n}}(J)=\left\{x \in \mathbb{R}^{n} \mid x_{j}=\operatorname{const}(j) \text { для некоторого } j \in J, x_{i}=\operatorname{const}(i) \text { для всех } i \notin J\right\},
$$

при этом, чтобы исключить вырожденные случаи, мы всегда предполагаем, что $k \geqslant 2$, т.е. $\operatorname{card} J \geqslant 2$ в определении $c_{\mathbb{R}^{n}}(J)$. Наконец, под кокрестом будем понимать любое множество $C \subset \mathbb{R}^{n}$ такое, что $C \subset c_{\mathbb{R}^{n}}(\{1, \ldots, n\} \backslash \operatorname{eqc}(C))$. (При этом всегда card eqc $(C)<n-1$.) На рис. 2 даны примеры кокрестов в $\mathbb{R}^{3}$.
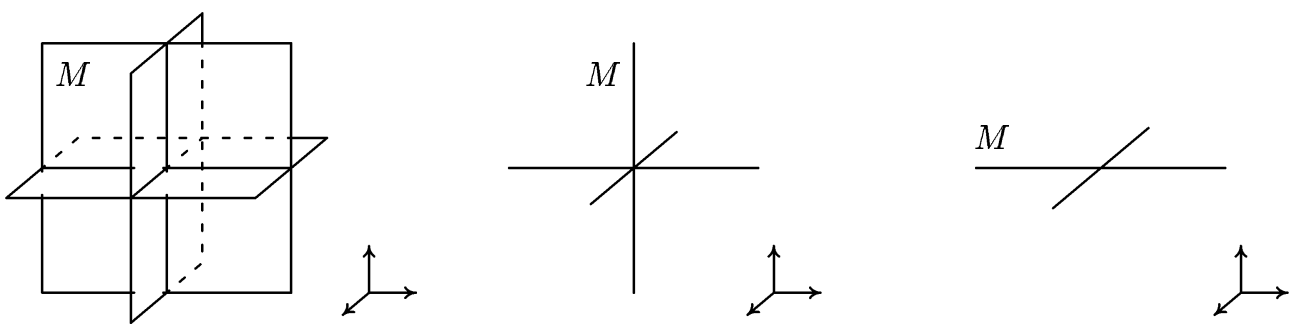

Рис. 2

Любое из множеств $M$, изображенных на рис. 2 , является кокрестом, равно как и подмножество $N \subset M$ c eqc $(N)=\operatorname{eqc}(M)$. Отметим, что первый и третий кокресты на рис. 2 не являются строго $\ell^{1}$-выпукльми множествами (хотя они $\ell^{1}$-выпуклы), а второй кокрест, определяемьй формулой $\left\{x=\left(x_{1}, x_{2}, x_{3}\right) \in \mathbb{R}^{3} \mid x_{i}=x_{j}=0\right.$ для некоторых $i \neq j\}$, является строго $\ell^{1}$-вьпуклым множеством.

Кокресты будут играть в некотором смысле исключительную роль в наших рассуждениях. Как мы увидим ниже (предложение 1), кокрест никогда не является строгим солнщем в $\ell^{\infty}(n)$.

В следующей основной теореме дается характеризация строгих солнц в $\ell^{\infty}(n)$.

Теорема. Пусть $\varnothing \neq M \subset \mathbb{R}^{n}$ замкнуто. Для того чтобы $M$ было строгим солнцем в $\ell^{\infty}(n)$, необходимо и достаточно, чтобы оно было строго $\ell^{1}$-выпукло и не являлось кокрестом.

1. Исторические замечания. Здесь и ниже $X$ - конечномерное нормированное пространство над $\mathbb{R}$.

Множество $M \subset X$ называется чебышёвским множеством, если для каждой точки $x \in X$ множество $P_{M} x$ одноточечно. Множество $M$ назьвается солнием, если для каждой точки $x \in X \backslash M$ существует точка $y \in P_{M} x$ такая, что $y \in P_{M}[(1-\lambda) y+\lambda x]$ для всех $\lambda \geqslant 0$. Ясно, что каждое строгое солнще является солнщем (обратное, вообще говоря, неверно, см. [1]; к примеру, любое множество (кокрест) на рис. 2 является солнцем в $\ell^{\infty}(n)$, но не является строгим солнцем). В конечномерном пространстве каждое чебьшёвское множество является солнщем [1].

Вопрос о характеризации солнц, строгих солнц и чебышёвских множеств рассматривался многими авторами (см. [5]-[10]). Этот вопрос тривиален для солнц и строгих 
солнц, если пространство $X$ гладко (т.е. в каждой точке единичной сферы пространства $X$ опорная гиперплоскость единственна): в гладких $X$ всякое солнце (и, следовательно, всякое строгое солнце и всякое чебышёвское множество в конечномерном случае) вьпукло (см. [1]).

Пространство $\ell^{\infty}(n)$ негладко; его единичный шар - $n$-мерньй куб.

Структура чебышёвских множеств и солнц на плоскости $\left(\mathbb{R}^{2},\|\cdot\|\right)$ с произвольной нормой хорошо изучена: чебышёвские множества были охарактеризованы П. Грубером [11], а солнца - Х. Беренсом, Л. Хетцельтом [12] и Хетцельтом [13]. Геометрическая характеризация строгих солнц на плоскости через опорные прямые, данная в [13], является ошибочной. Топологическая характеризация компактных чебышёвских множеств и компактных солнц на нормированной и несимметрично нормированной плоскости получена в [14].

В 1968 году в докторской диссертации [9] Б. Брозовский ввел понятие регулярного множества $M \subset C(Q), Q$ - компакт: $M$ регулярно, если для любых $x, x_{0} \in M$ и замкнутого множества $A \subset Q$ такого, что

$$
\inf _{q \in A}\left|x(q)-x_{0}(q)\right|>0
$$

элемент $x_{0}$ содержится в замыкании множества

$$
\left\{v \in M \mid\left(v(q)-x_{0}(q)\right) \cdot\left(x(q)-x_{0}(q)\right)>0 \text { для всех } q \in A\right\} .
$$

В этом случае пишем $\left(x, x_{0}\right) \in \operatorname{Reg}(M)$.

Это понятие было независимо введено Ч. Данемом (см. [8], где данное понятие детально исследуется и используется, а также обзор Браесса [7]).

Следующая теорема описьвает строгие солнща в $\ell^{\infty}(n)$.

Теорема А (Б. Брозовский [9], [10], [7], Ч. Данем [8]). Для непустого замкнутого подмножсества $M \subset \ell^{\infty}(n)$ следующие условия әквивалентны:

а) $M-$ строгое солние;

б) М регулярно.

Браесс [7] (см. лемму В ниже) установил свойство строгих солнц в $\ell^{\infty}(n)$, в некоторых случаях более сильное, чем $\ell^{1}$-вьпуклость, но более слабое, чем строгая $\ell^{1}$-вьпуклость (здесь мы пользуемся нашей терминологией).

Лемма В (Д. Браесс [7]). Пусть $M-$ строгое солние в $\ell^{\infty}(n)$. Eсли $u, w \in M$, то существует непрерывная дуга $k(t) \subset M, 0 \leqslant t \leqslant 1$, соединяющая и $c w$, такая, что ее координатные функиии $k_{i}(t)$ монотонны по $t, i=1, \ldots, n$. Функиии $k_{i}(t)$ строго монотонны при условии, что $u \neq w$.

Чебышёвские множества в $\ell^{\infty}(n)$ охарактеризованы Ч. Данемом [8] (см. также [7]).

Для солнц в $\ell^{\infty}(n)$ элегантная характеризация была получена Х. Беренсом и Л. Хетцельтом в [6] (см. также [5]). 
Теорема С (Х. Беренс, Л. Хетцельт [6]). Замкнутое непустое множество $M \subset \mathbb{R}^{n}$ является солнцем в $\ell^{\infty}(n)$, если и только если оно $\ell^{1}$-выпукло.

Как следствие из теоремы $\mathrm{C}$ в [6] получен важный результат, что солнце в $\ell^{\infty}(n)$ $V$-ациклично (т.е. его пересечение с любым замкнутым шаром ациклично и, следовательно, связно). Для произвольных негладких пространств $V$-связность и $V$-ацикличность солнц до сих пор не полностью исследованы (см. [5], [15]; к примеру, в конечномерном пространстве $V$-ацикличное множество является солнцем, но неизвестно, верно ли обратное). (Множество $V$-связно, если его пересечение с любым замкнутым шаром связно.) Солнца являются линейно связньци и локально линейно связными [2], [3], [5]. В.А. Кощеев [2] доказал, что в конечномерном линейном нормированном пространстве строгое солнце $V$-связно. Вопрос о $V$-связности солнц в таких пространствах размерности $\geqslant 3$ открыт. Также не известно, является ли строгое солнце в произвольном конечномерном пространстве $V$-ацикличньм.

2. Вспомогательные результаты. Напомним несколько нужных определений и результатов.

Если $X$ - линейное нормированноепространство и $x \in X, r>0$, то $B(x, r)$ и $\stackrel{\circ}{B}(x, r)-$ замкнутый и соответственно открытый шар с центром $x$ и радиусом $r$. Для $x, y, x \neq y$, опорньй конус к шару $B(x,\|x-y\|)$ в точке $y$ определяется как

$$
\stackrel{\circ}{K}(y, x)=\bigcup_{r \geqslant 0} \stackrel{\circ}{B}(-r y+(r+1) x,(r+1)\|x-y\|)
$$

(другие эквивалентные определения могут быть найдены в $[1$, гл. 3$]$ ).

Следующая лемма (см., например, $[16],[17],[1$, гл. 3]) является аналогом известного критерия Колмогорова. В [16] лемма доказана также для $\alpha$-солнц.

Лемма D (А. Брондстед [17], Е. В. Ошман [16]). 1. Множество $M \subset X$ является солнием в $X$ тогда и только тогда, когда для каждой точки $x \notin M$ найдется точка $y \in P_{M} x$ такая, что $K(y, x) \cap M=\varnothing$.

2. Множество $M \subset X$ является строгим солнцем в $X$ тогда и только тогда, когда для кажддой точки $x \notin M$ и для любой точки $y \in P_{M} x \neq \varnothing$ выполнено $\stackrel{\circ}{K}(y, x) \cap M=\varnothing$.

Из теоремы А следует важное следствие. Прежде чем его сформулировать, дадим два определения. Собственное аффинное подпространство $H$ в $\mathbb{R}^{n}$ назовем координатныц $\mathcal{M}$, если найдутся точка $x \in \mathbb{R}^{n}$ и число $r>0$ такие, что $H=\operatorname{aff} E$, где $E$ - некоторая грань шара $B(x, r)$ в пространстве $\ell^{\infty}(n)$. Иньми словами, $H=\left\{x \in \mathbb{R}^{n} \mid x_{j}=\operatorname{const}(j)\right.$ для любого $j \in \operatorname{eqc}(H) \neq \varnothing\}$.

Если $H$ - координатное афинное подпространство в $\mathbb{R}^{n}$ и $M \subset \mathbb{R}^{n}$, то определим

$$
M_{H}=\operatorname{pr}_{H} M \text { - координатная проекция } M \text { на } H,
$$

т.е. для $x \in M \operatorname{pr}_{H}(x)=\left(x_{1}^{\prime}, \ldots, x_{n}^{\prime}\right)$, где $x_{i}^{\prime}=x_{i}$ при $i \notin \operatorname{eqc}(H)$ и $x_{i}=h_{i}$ для $i \in \operatorname{eqc}(H)$ и любого $h \in H$.

СлЕДСТвИЕ 1. Пусть $M$ - строгое солние в $\ell^{\infty}(n), H$ - координатное аффинное подпространство в $\mathbb{R}^{n}$. Тогда $M_{H}$ - регулярное множество в $\left(H,\|\cdot\|_{\infty}\right)$.

Здесь норма $\|\cdot\|_{\infty}$ на $H$ индуцирована $\ell^{\infty}$-нормой на $\mathbb{R}^{n}$. 
ДокАЗАТЕЛЬСТво. Пусть $x, y \in M_{H}, x \neq y$, и пусть $\xi, \eta \in M$ таковы, что $\operatorname{pr}_{H} \xi=x$, $\operatorname{pr}_{H} \eta=y$. По теореме $\mathrm{A}(\xi, \eta) \in \operatorname{Reg}(M)$. Это означает, что для любого набора индексов $I \subset\{1, \ldots, n\} \backslash \operatorname{eqc}(\xi, \eta)$ выполнено

$$
\eta \in \operatorname{cl}\left\{\zeta \in M \mid\left(\zeta_{i}-\eta_{i}\right)\left(\xi_{i}-\eta_{i}\right)>0 \text { для всех } i \in I\right\} .
$$

Докажем, что $(x, y) \in \operatorname{Reg} M_{H}$. Пусть $J \subset\{1, \ldots, n\} \backslash \operatorname{eqc}(x, y)$. Ясно, что $J \subset I$. Отсюда

$$
y=\operatorname{pr}_{H} \eta \in \operatorname{cl}\left\{z \in M_{H} \mid\left(z_{i}-y_{i}\right)\left(x_{i}-y_{i}\right)>0 \text { для всех } i \in J\right\},
$$

т.е. $(x, y) \in \operatorname{Reg} M_{H}$, откуда по определению $M_{H}$ регулярно в $\left(H,\|\cdot\|_{\infty}\right)$.

Отметим, что если $M_{H}$ замкнуто, то по теореме А оно является строгим солнцем в $\left(H,\|\cdot\|_{\infty}\right)$.

Построим пример строгого солнца $M$ в $\ell^{1}(3)$ такого, что замыкание его проекции на $H=\mathbb{R}^{2}$ не будет являться строгим солнцем в $\ell^{\infty}(2)$ (хотя сама проекция $M_{H}$ будет регулярньп множеством). На плоскости $\mathbb{R}^{2}$ для каждого $t \in \mathbb{R}$ зададим ломаную $M_{t}$ следующим уравнением в полярной системе координат $(r, \varphi)$ :

$$
M_{t}=\left\{r \geqslant 0, \varphi=-\frac{1}{4} \operatorname{arcctg} t\right\} \cup\left\{r \geqslant 0, \varphi=\frac{\pi}{2}+\frac{1}{4} \operatorname{arcctg} t\right\} .
$$

По теореме А множество $M=\bigcup\left\{\left(M_{t}+(0,0, t)\right) \mid t \in \mathbb{R}\right\}$ является строгим солнцем в $\ell^{\infty}(3)$, однако его проекция на $\mathbb{R}^{2}$ представляет собой регулярное множество

$$
\left\{\left(x_{1}, x_{2}\right) \in \mathbb{R}^{2} \mid x_{1} \cdot x_{2}<0\right\} \cup\{(0,0)\},
$$

замькание которого не является строгим солнцем в $\ell^{1}(2)$.

Используя следствие 1 , мы докажем следующее интуитивно ясное полезное в дальнейшем утверждение.

ПРЕДЛОЖЕНИЕ 1. Никакой кокрест не является строгим солнцем в $\ell^{\infty}(n)$.

ДокАЗАТЕЛЬСТво. Пусть $C$ - кокрест в $\mathbb{R}^{n}$. Без ограничения общности считаем, что $\operatorname{eqc}(C)=\varnothing$.

Предположим, что $C$ - строгое солнце в $\ell^{\infty}(n)$.

Не ограничивая общности, будем считать, что const $(j)=0$ для любого $j$ в определении $c\left(\mathbb{R}^{n}\right) \supset C$, т.е. для любой точки $x \in C$ найдется индекс $i \in\{1, \ldots, n\}$ такой, что $x_{i}=0$.

1. Предположим, что $C$ содержит точку $c=\left(c_{1}, \ldots, c_{n}\right)$, у которой лиш одна координата $c_{i_{0}}$ равна нулю. Поскольку $C \subset c\left(\mathbb{R}^{n}\right)$, то несложно видеть, что

$$
\begin{gathered}
c \in P_{C} e_{k}, \quad \text { где } k= \pm 1, \quad e_{k}=\left(\varepsilon_{1} R, \ldots, \varepsilon_{n} R\right), \quad R=\max _{i=1, \ldots, n}\left\{\left|c_{i}\right|\right\}, \\
\varepsilon_{i}=\operatorname{sign} c_{i}, \quad i=1, \ldots, n, \quad i \neq i_{0}, \quad \varepsilon_{i_{0}}=k .
\end{gathered}
$$

По предположению $C$ - строгое солнще. Однако здесь для удобства дальнейших рассуждений мы воспользуемся более слабьм, нежели строгая солнечность, свойством множества $C$ - регулярностью, которой обладает множество $C$ по теореме А. В [10] доказано, что

если $M$ - регулярное множество в $\ell^{\infty}(n), x \notin M, y \in P_{M} x$, то $\stackrel{\circ}{K}(y, x) \cap M=\varnothing$. 
В нашей ситуации $c \in P_{C} e_{k}, k= \pm 1$, поэтому согласно $(1) \stackrel{\circ}{K}\left(c, e_{k}\right) \cap C=\varnothing, k= \pm 1$. По построению размерность грани шара $B\left(e_{k}, R\right)$, содержащей точку $c$ в своей относительной внутренности, равна $n-1, k= \pm 1$. Из этого следует, что конусы $\stackrel{\circ}{K}\left(c, e_{k}\right)$, $k= \pm 1$, являются взаимно дополняющими друг друга открытыми полупространствами с общей гранищей $G$, являющейся аффинньм координатным подпространством. Так как $\stackrel{\circ}{K}\left(c, e_{k}\right) \cap C=\varnothing, k= \pm 1$, то $C \subset G$ и еqс $(C) \supsetneqq \operatorname{eqc}(G)$. Но $G$ - координатное подпространство, т.е. $\operatorname{eqc}(G) \neq \varnothing$, что противоречит предположению еqс $(C)=\varnothing$. Случай 1 невозможен.

2. Предположим, что $n \geqslant 3$. Предположим также, что, в отличие от п. 1 , каждая точка из $C$ имеет не менее двух нулевых координат.

Спроектируем $C$ на координатное подпространство $\mathbb{R}^{n-1}:=\left\{x \in \mathbb{R}^{n} \mid x_{n}=0\right\}:$

$$
C_{n-1}=\operatorname{pr}_{\mathbb{R}^{n-1}} C \text {. }
$$

Так как еqс $\mathbb{R}^{n} C=\varnothing$, то еqс $\mathbb{R}^{n-1}\left(C_{n-1}\right)=\varnothing$. Согласно следствию $1 C_{n-1}-$ регулярное множество в пространстве $\ell^{\infty}(n-1)$. По предположению каждая точка из $C$ имеет не менее двух нулевых координат, поэтому $C_{n-1} \subset c\left(\mathbb{R}^{n-1}\right)$, а поскольку еqс $_{\mathbb{R}^{n-1}}\left(\mathrm{C}_{n-1}\right)=\varnothing$, то $C_{n-1}-$ кокрест в пространстве $\mathbb{R}^{n-1}$.

3. Далее рассуждаем аналогично предыдущему. Если найдется точка $c \in C_{n-1} \mathrm{c}$ только одной ненулевой координатой (в пространстве $\mathbb{R}^{n-1}$ ), то мы оказьваемся в ситуации п. $1 \mathrm{c} \mathbb{R}^{n}$, замененньм на $\mathbb{R}^{n-1}$, и рассуждая аналогично, приходим к противоречию. Если же $n-1 \geqslant 3$ и все точки из $C_{n-1}$ имеют не менее двух нулевых координат, то, действуя как в п. 2, уменьшаем размерность на единицу. В результате мы или придем к противоречию, или к ситуации $n=2, C_{2} \subset c\left(\mathbb{R}^{2}\right)-$ кокрест в $\mathbb{R}^{2}$ и $C_{2}$ регулярно в $\ell^{\infty}(2)$.

Тогда в $C_{2}$ в силу условия еqс $\mathbb{R}^{2}\left(C_{2}\right) \neq \varnothing$ найдется точка с координатами $(\alpha, 0)$, $\alpha \neq 0$, а в силу п. 1 такая ситуация невозможна. Предложение доказано.

3. Основная теорема. В этом пункте мы доказываем основную теорему, которая усиливает лемму В и характеризует строгие солнца в $\ell^{\infty}(n)$. В сравнении с теоремой $\mathrm{A}$ здесь мы получаем чисто геометрическую характеризацию.

Теорема. Пусть $\varnothing \neq M \subset \mathbb{R}^{n}$ замкнуто. Для того чтобы $M$ было строгим солнием в $\ell^{\infty}(n)$, необходимо и достаточно, чтобы оно было строго $\ell^{1}$-выпукло и не являлось кокрестом.

ДокАЗАТЕЛЬСТво. Необходимость. Предположим, что $M-$ строгое солнце в $\ell^{\infty}(n)$. По предложению 1 множество $M$ не является кокрестом.

$\operatorname{Ecли~еqс~}(M)=\varnothing$, то отношение ' $\neq_{M}$ ' совпадает с ' $\neq$ ' и строгая $\ell{ }^{1}$-выпуклость $M$ немедленно следует из леммы В и теоремы С.

Предположим, что $\operatorname{eqc}(M) \neq \varnothing$; без ограничения общности предположим, что $\operatorname{eqc}(M)=\{1, \ldots, m\}, 1 \leqslant m<n$. Тогда $M$ содержится в аффинном координатном подпространстве $H_{n-m}$ размерности $n-m$. Следовательно, $H_{n-m}$ можно отождествить с пространством $\ell^{\infty}(n-m)$ (здесь мы без ограничения общности предполагаем, что $0 \in M)$. Ясно, что $M-$ строгое солнце в $\ell^{\infty}(n-m)$. Теперь строгая $\ell^{1}$-вьпуклость $M$ следует из леммы В и теоремы С, примененных к $\ell^{\infty}(n-m)$.

Достаточность. Достаточно считать, что еqс $(M)=\varnothing$. Тогда случай $\operatorname{eqc}(M) \neq \varnothing$ будет следовать из случая $\operatorname{eqc}(M)=\varnothing$, поскольку если eqc $(M) \neq \varnothing$ и $H$ - координатное 
аффинное подпространство в $\mathbb{R}^{n}$, содержашее $M$, и $M-$ строгое солнще в $\left(H,\|\cdot\|_{\infty}\right)$, то $M-$ строгое солнце в $\ell^{\infty}(n)$. Этот факт просто следует из теоремы А.

Итак, eqc $(M)=\varnothing$. По теореме С множество $M$ является солнцем. Докажем, что $M$ является строгим солнцем, для чего воспользуемся леммой $\mathrm{D}$ и докажем, что для любой точки $x \notin M$ и любой точки $y \in P_{M} x$ выполнено $\stackrel{\circ}{K}(y, x) \cap M=\varnothing$. Без ограничения общности считаем $x=0, \rho(0, M)=\|y\|=1$, а также $y_{i} \geqslant 0, i=1, \ldots, n$. Обозначим $J=\left\{j \mid y_{j}=1\right\}$. Так как $\|y\|=1$ и $y_{i} \geqslant 0$, то $J \neq \varnothing$.

Предположим, что $\stackrel{\circ}{K}(y, 0) \cap M \neq \varnothing$.

Для точки $v \in \mathbb{R}^{n}$ определим

$$
\mathscr{E}(v)=\left\{x \in \mathbb{R}^{n} \mid x_{m}=v_{m} \text { для некоторого } m=m(x) \in\{1, \ldots, n\}\right\} .
$$

В наших терминах $\mathscr{E}(v)$ - основной кокрест в $\mathbb{R}^{n}$. Определим $H=\mathscr{E}(y) \cap \stackrel{\circ}{K}(y, 0)$.

1. Пусть $w \in \stackrel{ }{K}(y, 0) \cap M$ и $w \notin H$. Тогда $w \notin \mathscr{E}(y)$. Поэтому $w \neq y$ и по строгой $\ell^{1}$-вьпуклости $M$ это влечет, что $w$ и $y$ могут быть соединены строго монотонньм $\ell^{1}$-геодезическим сегментом $k(t) \subset M, k(0)=y, k(1)=w$.

Докажем, что $k(t) \subset \stackrel{\circ}{B}(0,1)$ при всех достаточно малых $t$. В нашей ситуации $\stackrel{\circ}{K}(y, 0)=$ $\left\{z \mid z_{j}<1\right.$ при $\left.j \in J\right\}$. Так как $w \in \stackrel{\circ}{K}(y, 0)$ и координатные функции кривой $k(t)$ строго монотонны, то $w_{j}<k_{j}(t)<1$ при $j \in J$ и всех $0<t<1$. Поскольку $k_{j}(t) \rightarrow y_{j}=1$ при $t \rightarrow 0, j \in J$, то для некоторого $\varepsilon>0$

$$
0<k_{j}(t)<1 \quad \text { при } j \in J \text { и } t \in(0, \varepsilon) \text {. }
$$

Далее, $k_{m}(t) \rightarrow y_{m}$ при $t \rightarrow 0,1 \leqslant m \leqslant n$, и $0 \leqslant y_{i}<1$ при $i \notin J$. Поэтому при некотором $\varepsilon_{1}>0$

$$
\left|k_{i}(t)-y_{i}\right|<1-y_{i} \quad \text { при } i \notin J, 0<t<\varepsilon_{1} .
$$

Отсюда и из (2) имеем

$$
-1<k_{m}(t)<1 \quad \text { при } t \in\left(0, \min \left\{\varepsilon, \varepsilon_{1}\right\}\right), m=1, \ldots, n .
$$

Следовательно, $k(t) \subset \stackrel{\circ}{B}(0,1)$ при $t \in\left(0, \min \left\{\varepsilon, \varepsilon_{1}\right\}\right)$. Однако это невозможно, так как по условию $y \in P_{M} 0$, т.е. $\stackrel{\circ}{B}(0,1) \cap M=\varnothing$.

2. Предположим теперь, что $M \cap \stackrel{\circ}{K}(y, 0) \subset H$. Пусть $u \in H \cap M$. Так как $M$ не кокрест и еqс $(M)=\varnothing$, то $M \not \subset \mathscr{E}(u)$. Следовательно, найдется точка $v \in M, v \notin \mathscr{E}(u)$, т.е. $v \neq u$. В этом случае по строгой $\ell^{1}$-выпуклости $M$ точки $u$ и $v$ соединены строго монотонньм $\ell^{1}$-геодезическим сегментом $k(t) \subset M, 0 \leqslant t \leqslant 1, k(0)=u, k(1)=v$. Так как $u \in \stackrel{\circ}{K}(y, 0)$, то $u_{j}<1$ при $j \in J$ и, следовательно, $k_{j}(t)<1$ при $j \in J$ и $0<t<\varepsilon_{2}$ при некотором $\varepsilon_{2}$, т.е. $k(t) \in \stackrel{\circ}{K}(y, 0)$ при $0<t<\varepsilon_{2}$. Из строгой монотонности $k(t)$ следует, что $k(t) \neq y$ при всех $t \in\left(0, \varepsilon_{3}\right)$ при некотором $\varepsilon_{3}$. Итак, при $0<t<\min \left\{\varepsilon_{2}, \varepsilon_{3}\right\}$ кривая $k(t) \subset M$ содержится в $\stackrel{\circ}{K}(y, 0)$ и не содержится в $H$, что противоречит предположению $M \cap \stackrel{\circ}{K}(y, 0)=H$.

Итак, предположение $\stackrel{\circ}{K}(y, 0) \cap M \neq \varnothing$ неверно и $M$ - строгое солнще по лемме D. Теорема доказана.

Автор благодарит рецензента за полезные замечания. Автор также благодарит Математический институт университета Эрланген-Нюрнберг и профессора Х. Беренса за гостеприимство в Эрлангене. 


\section{СПИСОК ЦИТИРОВАННОЙ ЛИТЕРАТУРЫ}

[1] Власов Л. П. Аппроксимативные свойства множеств в линейных нормированных пространствах // УМН. 1973. Т. 28. №6. С. 1-66.

[2] Кощеев В.А. Связность и аппроксимативные свойства множеств в линейных нормированных пространствах // Матем. заметки. 1975. Т. 17. № 2. С. 193-204.

[3] Кощеев В. А. Связность и солнечные свойства множеств в линейных нормированных пространствах // Матем. заметки. 1976. Т. 19. № 2. С. 267-278.

[4] Menger K. Untersuchungen über allgemeine Metrik // Math. Ann. 1928. V. 100. P. 75-163.

[5] Brown A. L. Suns in normed linear spaces which are finite-dimensional // Math. Ann. 1987. V. 279. P. 81-101.

[6] Berens H., Hetzelt L. Die Metrische Struktur der Sonnen in $\ell^{\infty}(n) / /$ Aequat. Math. 1984. V. 27. P. 274-287.

[7] Braess D. Geometrical characterizations for nonlinear uniform approximation // J. Approximation Theory. 1974. V. 11. P. 260-274.

[8] Dunham Ch. B. Characterizability and uniqueness in real Chebyshev approximation // J. Approximation Theory. 1969. V. 2. P. 374-383.

[9] Brosowski B. Nich-Lineare Tschebyscheff-Approximation. B. I. Hochschulskripten. V. 808/ 808a. Bibliographisches Institut, Mannheim: Hochschultaschenbücher Verlag, 1968.

[10] Brosowski B., Wegmann R. Charakterisierung bester Approximationen in normierten Räumen // J. Approximation Theory. 1970. V. 3. P. 369-397.

[11] Gruber P. M. Planar Chebyshev sets // Mathem. Structure-Computational Math. - Math. Modelling. V. 2. Sofia: Bulgar. Acad. Sci., 1984. P. 184-191.

[12] Berens H., Hetzelt L. Suns and contractive retracts in the plane // Теория приближений функций / ред. Н. П. Корнечук и др. Труды международной конференции (Киев, 31 мая-5 июня 1983г.). М.: Наука, 1987. С. 483-487.

[13] Hetzelt L. On suns and cosuns in finite-dimensional normed linear spaces // Acta Math. Hung. 1985. V. 45. P. 53-68.

[14] Алимов А. Р. Чебышёвские компакты на плоскости // Тр. МИАН. 1997. Т. 219. С. 8-26.

[15] Карлов М. И., Царьков И. Г. Выпуклость и связность чебышёвских множеств и солнц // Фундамент. и прикл. матем. 1997. Т. 3. № 4. С. 967-978.

[16] Ошман Е.В. Чебышёвские множества и непрерывность метрической проекции // Изв. вузов. Матем. 1970. Т. 9. С. 78-82.

[17] Brøndsted A. Chebyshev sets and convexity of Chebyshev sets. II // Math. Scand. 1966. V. 18. P. 5-15. 